\title{
The Scientific Calculator and School Mathematics
}

\author{
Barry Kissane \\ Murdoch University Western Australia \\ $<$ b.kissane@murdoch.edu.au>
}

\begin{abstract}
Scientific calculators are sometimes regarded as important only for obtaining numerical answers to computational questions, and thus in some countries regarded as inappropriate for school mathematics, lest they might undermine the school curriculum. This paper argues a contrary view that, firstly, numerical computation is not the principal purpose of scientific calculators in education, and secondly that calculators can play a valuable role in supporting students' learning. Recent developments of calculators are outlined, noting that their principal intention has been to make calculators easier to use, align their functionality with the school mathematics curriculum and represent mathematical expressions in conventional ways. A model for the educational use of calculators is described, with four key components: representation, computation, exploration and affirmation. Examples of how these might impact positively on school mathematics are presented, and suggestions are made regarding good pedagogy and curriculum with calculators in mind. The paper concludes that scientific calculators represent the best available technology to provide widespread access to some ICT in the mathematics curriculum for all students in the SEAMEO region.
\end{abstract}

Keywords: scientific calculator, learning, technology, curriculum, assessment

\section{Introduction}

We live in an age of technology. Throughout much of Indonesia and other countries in the SEAMEO region there is now widespread evidence of the everyday use of technology, especially in urban areas. Smart phones are almost universal; businesses routinely use computers and many students have developed expertise with these and other forms of technology. The exception to these kinds of generalisations are schools and in particular the mathematics curriculum in schools, which often appears to be impervious to technological change. In this paper we explore the potential for scientific calculators to provide a helpful injection of technology for learning mathematics into secondary schools.

Information and Communications Technology (ICT) is widely recognized as an important influence on school mathematics at least in western countries, but also increasingly in other countries, including SEAMEO countries. For example, the National Council of Teachers of Mathematics (NCTM, 2015) in the USA espouses a Technology Principle as one of its core principles for the mathematics curriculum: "Technology is essential in teaching and learning mathematics; it influences the mathematics that is taught and enhances students' learning." 
The NCTM has suggested the technology might change not only how teachers teach and how students learn but also the content of the mathematics curriculum. It might change what is regarded as important. It will provide a different perspective on the appropriate emphasis of the curriculum. The first requirement of technology having these kinds of effects is that it is available to students. A significant advantage of the calculator over other forms of technology is that it is relatively affordable, especially in comparison with other forms of technology, as argued by Kissane and Kemp (2012). For many students in affluent societies or indeed for many affluent families in other societies, technology is routinely available but that's not the case for all families in all societies, and seems unlikely to be the case for students and their families across the SEAMEO region.

In this regard, the recent OECD (2015) study provides some useful data regarding participating SEAMEO countries; the study investigated computer availability and Internet use, but nonetheless gives a sense of access to these forms of ICT in some countries. (Like most studies of ICT and policy documents, calculators were not included, presumably because they are not regarded as a form of technology relevant to schools.) Table 1 shows that, with the exceptions of Singapore and Thailand, SEAMEO countries had substantially more students per computer than the OECD average. Only four of the 64 countries reported in the study had a higher ratio of students to school computers, suggesting that access to ICT in these three countries is relatively very limited.

Table 1.

Number of students per computer in SEAMEO countries in 2012

\begin{tabular}{ll}
\hline Singapore & 2.0 \\
Thailand & 3.1 \\
OECD average & 4.7 \\
Vietnam & 8.6 \\
Indonesia & 16.4 \\
Malaysia & 16.7 \\
\hline
\end{tabular}

Source: OECD (2015, pp 20-21)

The OECD study also examined home access to computers, reporting the percentage of students with at least one computer at home. Table 2 displays the reported data. 
Table 2.

Percentage of students in SEAMEO countries with at least one computer at home in 2012

\begin{tabular}{ll}
\hline Singapore & 96.9 \\
OECD average & 95.8 \\
Malaysia & 74.0 \\
Thailand & 65.6 \\
Vietnam & 38.9 \\
Indonesia & 25.8 \\
\hline
\end{tabular}

Source: OECD (2015, pp 18-19)

Although the figures in Table 2 suggest that home computers are relatively available to students in the participating SEAMEO countries, most noticeably Singapore, a sense of perspective is provided by the observation that the other four SEAMEO countries listed are amongst the 12 participating countries in which a home computer is least prevalent; furthermore, of the 64 countries studied, Vietnam and Indonesia are the two countries with the lowest percentages of students with at least one computer at home. These data, albeit not directly concerned with calculators, suggest that computer use in schools and at homes is less prevalent in these SEAMEO countries than elsewhere (Singapore excepted). It seems likely, too, that the distribution of computers, both at homes and in schools, is likely to be uneven within many SEAMEO countries, especially across urban and rural areas. Together, these data, despite their limitations, suggest that consideration of alternative forms of technology for mathematics, scientific calculators in particular, may be warranted.

Calculators have been around now for some forty years or so, and are in widespread use within schools in many countries. When they were first introduced in schools, some teachers and others were concerned that they may detract from students learning mathematics, but extensive research into that sort of question has reached a consistent conclusion that this is not the case. In a recent meta-analysis review of a large body of research, Ronau et al., (2011) observed, with some evident frustration:

Few areas in mathematics education technology have had such focused attention with such consistent results, yet the issue of whether the use of calculators is a positive addition to the mathematics classroom is still questioned in many areas of the mathematics community, as evidenced by continually repeated studies of the same topic. As a result, we concluded that future practitioner questions about calculator use for mathematics teaching and learning should advance from questions of whether or not they are effective to questions of what effective practices with calculators entail. (Ronau et al, 2011, p.2) 
This paper offers suggestions regarding the effective use of scientific calculators, which seems to have been frequently overlooked as potential learning and teaching tools. The paper first clarifies the nature of scientific calculators, highlighting how they have changed over time, and then presents a model for thinking about the potential benefits of their use in secondary schools. Finally, the paper makes some observations about the environment for use of technology in schools, especially the need for adequate support for teachers and a curriculum that has been designed to accommodate some use of technology.

\section{What is a Scientific Calculator?}

The scientific calculator first appeared in the 1970s. Early models were relatively primitive and even relatively expensive by the standards of today. Yet they quickly made their way into schools in many countries, especially for students learning mathematics. The early calculators were called 'scientific' presumably because they met the immediate and everyday computational needs of scientists (and engineers); the name is still used today. What distinguished a scientific calculator was not only its capacity to undertake arithmetic but also its capacity to retrieve values that were normally only available through a published table book. So a scientific calculator became immediately recognizable because its keyboard included trigonometric functions, logarithmic functions and keys for previously difficult tasks such as finding roots and powers of numbers. These characteristics are still evident in today's scientific calculators so that a casual glance might give the impression that not much has changed since the 1970s and is not surprising that many people still regard them as essentially devices for undertaking otherwise awkward and tedious computations.

While this is not an unreasonable view to hold, especially if someone has limited experience with a modern scientific calculator, it is an incorrect view. Since the 1970s scientific calculators have been progressively improved and developed almost entirely for the purposes of secondary school mathematics, rather than to better meet the needs of the scientists and engineers for whom they were originally designed. The developments are of several kinds. In the first place scientific calculators have become much easier to use than they were originally. While they were never difficult to use, especially for scientists and engineers, secondary school students sometimes found the calculator interface more problematic. For example, when students put expressions into their calculator, the expressions disappeared as soon as the answer appeared so they were not sure what they had done. Similarly the way in which expressions were entered were different from the way in which students would normally think of them. As 
illustrations of this, to find the square root of a number, students first entered the number into their calculator and then tapped a square root key, or to find the cosine of an angle they firstly entered the angle and then tapped the cosine key. Of course, students learned to do such things, but having to do so made using the calculator a more difficult device then would be preferable and indeed students had to learn how to use the calculator as well as how to understand the mathematical ideas that were involved. Modern calculators are much more user-friendly then their ancient counterparts of the 1970s, with students able to see exactly what has been entered, able to edit expressions, able to see and recall previous results easily, and able to recall information (such as statistical data) for verification.

A second major development of scientific calculators concerns the aspects of mathematics with which they deal. As well as becoming easier to use in general, modern scientific calculators such as the CASIO Fx-991 ID PLUS, provide students with the capability to address many aspects of mathematics important to the school curriculum. Elementary examples are the routine use of fractions as well as decimals, the use of complex numbers where necessary and the use of vectors and matrices to support advanced mathematical work. But there are more sophisticated examples than these, never imagined when the first scientific calculators were produced. Modern scientific calculators allow students to tabulate functions over a domain rather than merely to evaluate a single value of the function. They allow students to enter and store empirical data and analyse the data in a variety of ways. They allow students to solve elementary equations such as quadratic and cubic equations, as well as systems of linear equations and more sophisticated equations. They allow students to find numerical derivatives and numerical definite integrals. Functionality of these kinds has been deliberately designed into modern calculators to improve the extent to which they match the mathematics curriculum in schools; they have not been designed to meet the needs of scientists and engineers, who are probably more likely to use sophisticated computer technology much of the time these days.

In addition to becoming user friendly and addressing more aspects of mathematics, modern scientific calculators represent quantitative expressions in the same way in which they routinely appear in textbooks, on blackboards and in everyday handwriting. There are many examples of this including representing fractions in the conventional way, using radical signs correctly, using a smaller font for indices and powers, and using integration and summation symbols conventionally. Later parts of this paper show several examples of these sorts of representations. As well as appearing familiar to students, natural displays of these kinds mean 
that students do not need to learn complex rules for how to enter and interpret information on a calculator screen. The calculator is designed to be part of the mathematical environment of students learning mathematics in school, representing mathematical operations in the same way as the other media which students are interacting.

As well as these improvements, the scientific calculator has a number of attributes which make it particularly valuable for use in developing countries as suggested by Kissane and Kemp (2012). Perhaps the most obvious of these concerned the relative affordability of calculators, their physical size and their lack of need of sophisticated technological infrastructure. Because calculators cost so much less then devices like computers or tablets, they are much more likely to be available to individual students on a wide scale. Because calculators are physically small and quite robust they are easily transported by students from class to class and from class to home so that they provide individual student access to technology in a variety of settings. Unlike computer-based forms of technology, calculators do not require an IT department, hardware and software upgrades, telecommunications capabilities, or even a regular electricity supply. Also unlike computer-based forms of technology, scientific calculators are rarely a source of distraction, as students cannot use them for web browsing, social media and other activities unrelated to education. In many parts of the SEAMEO region, these various attributes render calculators accessible to all students rather than to only the most affluent students or the most affluent schools, and offering significant potential for educational use, as the following section describes.

\section{Educational Use}

It seems not unusual for people to regard calculators as solely useful for numerical computation. Were this impression correct, however, an argument for using calculators in secondary school would be relatively limited, as mathematics involves a great deal more than numerical computation. As Kissane and Kemp (2014) have argued, the educational case involves much more than facilitating computation. They suggested a model for calculator use in education that involved four different components, respectively representation, computation, exploration, and affirmation. In this section, some examples of these four aspects are provided and briefly discussed, using the CASIO fx-991ID PLUS scientific calculator for that purpose. This particular calculator is designed for use in Indonesia; other calculators at the same level have similar capabilities, although there are small differences among various models. However the main points can be well illustrated efficiently by focusing on a particular model. 


\section{Representation}

Modern calculators use conventional mathematical syntax to represent various mathematical ideas, so that what students see on the screen is similar to what they might write on their page or see on blackboards and in textbooks. Figure 1 shows some examples of this, with expressions that were previously difficult for scientific calculators to represent conventionally. When the calculator uses the standard conventions, students can comfortably enter expressions as they would write them by hand, without having to learn a troublesome syntax.

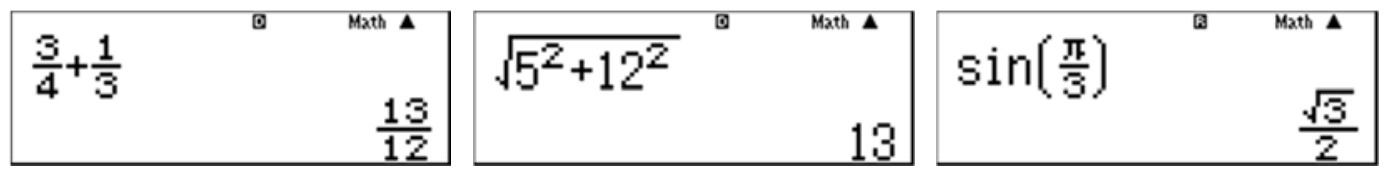

Figure 1. Representing and evaluating some mathematical expressions

More sophisticated representations are also available on modern calculators, reflecting the design of calculators for students in the senior secondary years, encountering more sophisticated ideas. Three examples of this are shown in Figure 2.

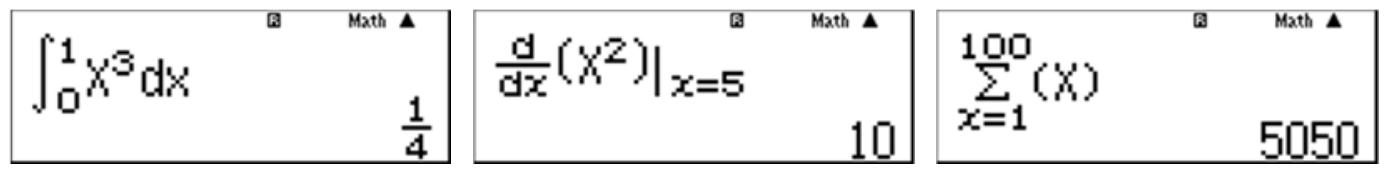

Figure 2. Representation of more sophisticated mathematical expressions

The idea of 'representation' is more subtle than merely displaying expressions in conventional mathematical ways, however. It might also be interpreted as a 're-presentation' or presenting something for a second time. Calculators do not necessarily produce as output what was input: in each of the cases in Figures 1 and 2, the output (on the bottom line of the display) differs from the input (on the first line of the display). Paying attention to these can have significant educational benefits for students. Figure 3 shows a good example, as the CASIO fx-991ID PLUS routinely represents non-integral numbers as fractions, when that is possible. In the first screen, students are given a clear indication of the meaning of a percentage by the re-presentation of the input of $37 \%$. The second screen arises from the use of a calculator key to toggle results between fractions and decimals, which in this instance offers students an opportunity to see a helpful decimal representation of a percentage. 


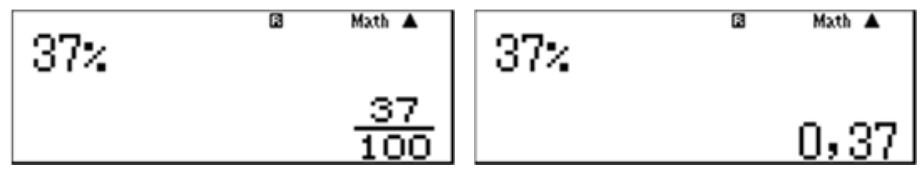

Figure 3. Re-presentation of a percentage

There are many other contexts in which a calculator will re-present mathematical expressions in ways that are at least conceptually helpful, although sometimes also challenging, but nonetheless educationally productive. Three examples are shown in Figure 4.
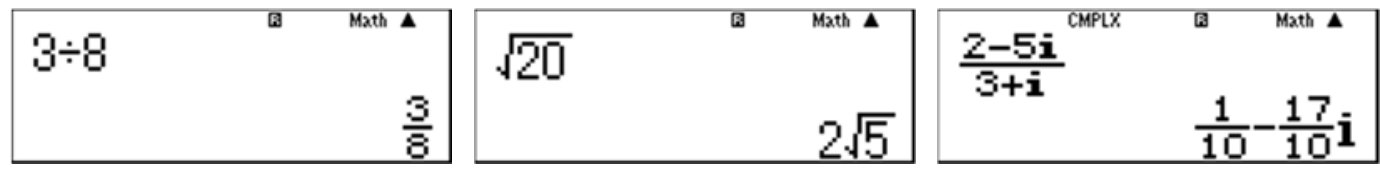

Figure 4. Further re-presentations of mathematical expressions

Although the results shown in Figure 4 can all be represented by the calculator as decimals, there are substantial conceptual advantages of the representations shown, both supporting and encouraging students to make sense of the ideas that are involved and the processes used.

Understanding key mathematical concepts, such as the concept of a function, are important for students learning mathematics. So, a calculator that represents the concepts in productive ways is a potentially important tool. Figure 5 shows how a calculator can represent the function $f(x)=x^{2}+3 x+2$ both symbolically and numerically in a table (which is shown only partially, with only three rows visible at once).
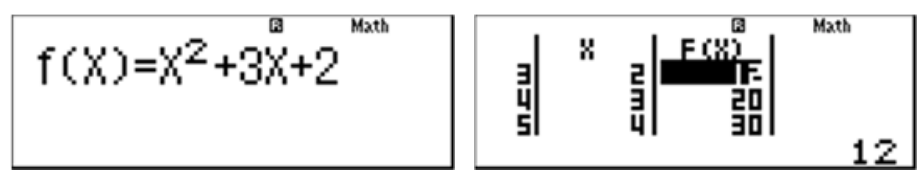

Figure 5. Representation of a function

From such representations, students can be supported to see yet other representations, such as $f(2)=12, f(3)=20$, etc. or as a set of ordered pairs, $\{\ldots,(2,12),(3,20),(4,30), \ldots\}$, or use the values given by the calculator to sketch a graph on paper.

\section{Computation}

Scientific calculators can handle any of the numerical computations that secondary school students are likely to encounter. Some parts of the mathematics curriculum require 
students to develop some personal expertise with computation, especially with mental computation and with sound approximations. A calculator should not be a substitute for developing expertise of those kinds. However, students who are deprived of calculator use have very little opportunity to make use of the mathematics they have learned in practical contexts, since the computations involved are frequently too difficult or tedious to complete without technological help.

Good examples of this involve the use of everyday measurement tasks and the use of real information. Textbooks often contain fictitious tasks such as finding the volume of a classroom that is $8 \mathrm{~m}$ wide, $10 \mathrm{~m}$ long and $3 \mathrm{~m}$ high; students with a calculator can be directed to find the actual volume of the room in which they are studying, after making their own measurements and be expected to deal appropriately with the practical issues of accuracy involved. They might also be asked to determine what size air conditioning unit would suit the room, and determine the cost of operating it for a school week. The experiences involved are quite different from each other, as the calculator can easily accommodate the awkward numbers involved, and help students to see that mathematics can be used in practice.

Similarly, students in Malaysia can be provided with real information regarding the population of Malaysia (which was 28334135 in the 2010 census) and real information about the population growth rate (which has been close to $1,5 \%$ per annum recently) and asked to use this information to predict the likely population in 2020. Figure 6 shows one of several ways of doing this, after storing the 2010 population into a variable memory, $A$.

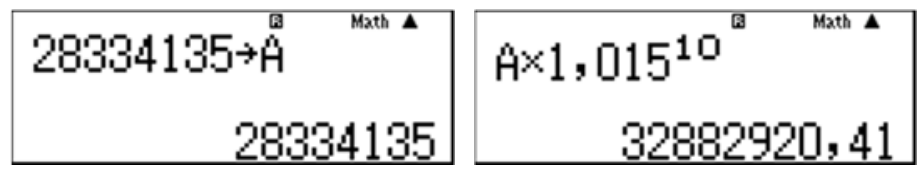

Figure 6. Predicting the population of Malaysia in 2020 to be 32882920

When the calculator has taken care of the computation, time is left for students to consider important aspects of mathematical modelling such as the reasonableness of an assumption of constant growth or the level of accuracy in the data and any predictions. Similarly, when computations are handled easily by the calculator, other computations can be considered, such as those showing in Figure 7, examining a sequence of population estimates for Malaysia under the different assumption that the population growth rate is reduced to 1,4\% per annum. In this case, the estimate of the population for 2020 is of course a little less than was the case in Figure 6. 

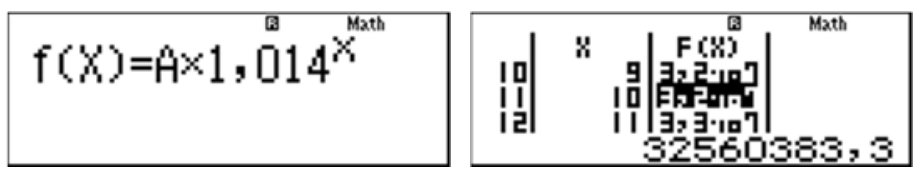

Figure 7. Predicting Malaysian populations under different assumptions

Real data are always awkward to handle computationally, so students without a calculator at their disposal are typically given artificial (and small) data sets, so that they any computations involved are manageable. When students have a scientific calculator, not only are they able to use real data from official or published sources (such as those shown in Figures 6 and 7), but they can actually engage in their own data collection and use real data that they have collected. This makes a profound difference to the nature of the statistics curriculum, which can involve students undertaking their own investigations rather than using fictitious data from a textbook.

Modern calculators, such as the CASIO fx-991ID PLUS, include a capacity for many kinds of inbuilt computations relevant to the school mathematics curriculum. Figure 8 shows a typical example, concerned with the solution of the quadratic equation, $x^{2}=x+1$. The type of equation is chosen from a suite of possibilities, and the coefficients entered in a conventional way (after some rearrangement into the necessary order of descending powers of $x$ ):
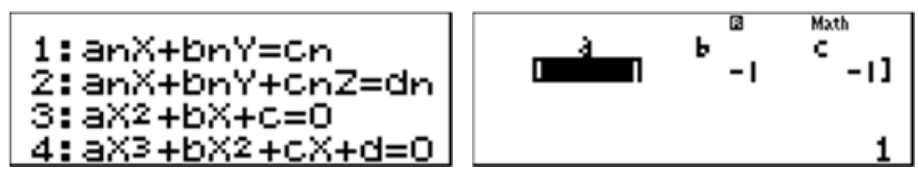

Figure 8. Entering the quadratic equation $x^{2}=x+1$ for solution

The solutions of the equation are then available via successive keystrokes and are shown in Figure 9. In addition, the calculator routinely computes the turning point of the associated parabola, as shown in Figure 10.

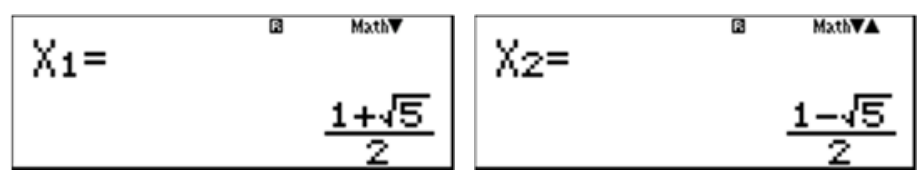

Figure 9. Solutions of $x 2=x+1$

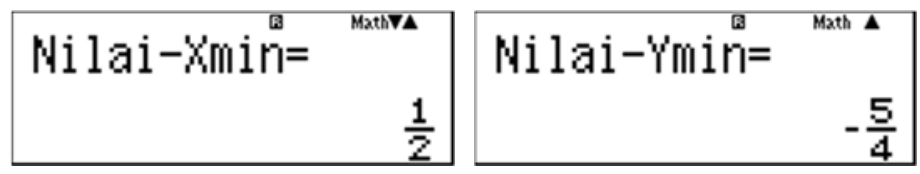

Figure 10. Computing the turning point of $f(x)=x^{2}-x-1$ 
Although the calculations in Figures 9 and 10 can be undertaken by hand, the procedures involved are routine and algorithmic in nature, and shed little light on the key mathematics involved. When the calculator is available to do the computations, students have some time and space left to consider the results, noticing in this case for example, the symmetry around the line $x=1 / 2$.

Finally, some aspects of mathematics are computationally intensive, although the computations involved provide little insight regarding the mathematical ideas involved. A good example involves the solution of a system of linear equations. Another involves the inversion of a matrix. To illustrate, consider the linear system:

$$
\begin{gathered}
x+2 y=5 \\
3 x-y=11
\end{gathered}
$$

Coefficients for this system can be entered into the calculator, and the solutions obtained with successive keystrokes, as shown in Figure 11.

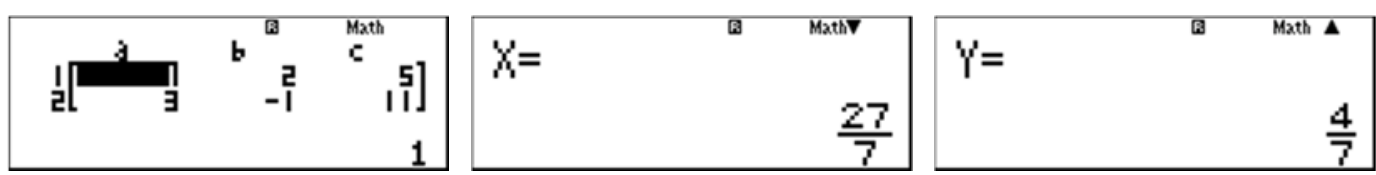

Figure 11. Solving a 2 × 2 linear system

Alternatively, the system can be represented with a matrix of coefficients $\boldsymbol{A}$ and a solution matrix $\boldsymbol{B}$, as shown in Figure 12.

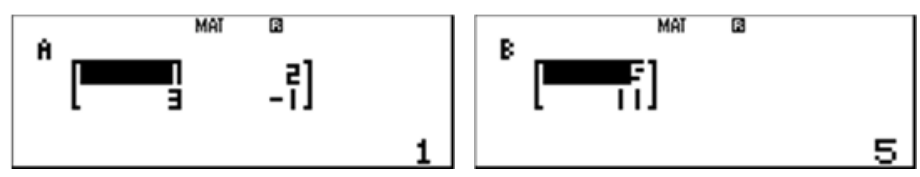

Figure 12. Representing the linear system, $A X=B$

The solution relies on computing $\boldsymbol{X}=\boldsymbol{A}^{-1} \boldsymbol{B}$, requiring the inverse of a matrix and matrix multiplications. Such computations are both tedious and error-prone, and better left to a calculator, as shown in Figure 13.
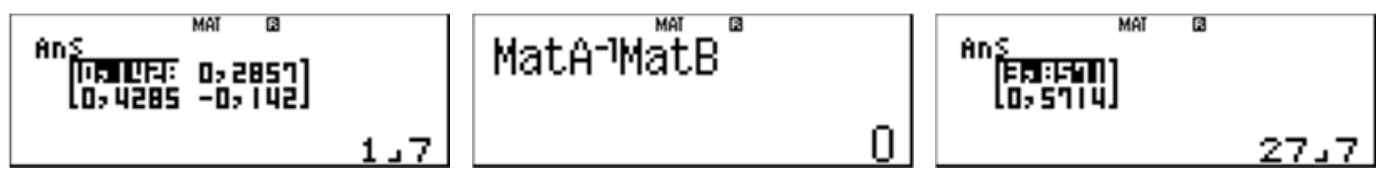

Figure 13. Matrix inversion (1st screen) and matrix solution (3rd screen) of the 2 × 2 system

Such computations allow students to see the important connections between matrices and the solutions of systems of equations, without being bogged down in tedious hand computations. 


\section{Exploration}

Access to a calculator allows students to explore mathematical situations for themselves, which can be an important avenue to learning. In effect, the calculator is an educational device that facilitates exploration, not just a device to obtain numerical answers. There are many examples of this sort of activity, but space here to describe briefly only a few, in increasing order of mathematical sophistication.

Young students first encountering different representations of numbers, in the form of fractions and decimals can make good use of a modern scientific calculator to understand how these various representations are related. Figure 14 shows some examples of this.

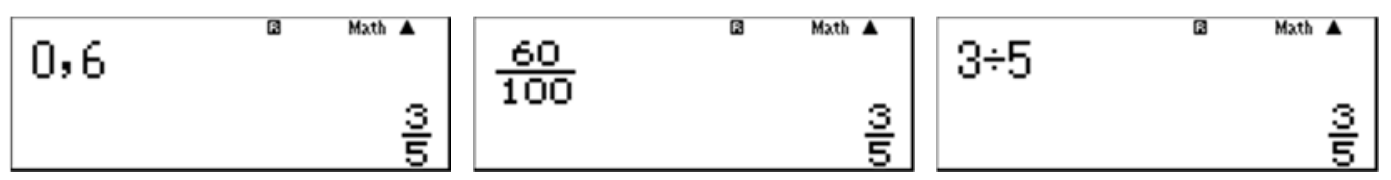

Figure 14. Different representations of three fifths

Explorations with a calculator can help students to see that fractions are numbers (not pairs of numbers, as students commonly think), can help them to see that many fractions are in fact the same number (through the idea of equivalent fractions) and that fractions can be interpreted in terms of divisions of integers. The natural operations of the calculator, together with the availability of a fractions to decimals key, provide a rich environment for explorations among these areas.

Earlier, Figure 5 showed how a calculator can represent a function numerically, as well as symbolically. Students in the early years of studying algebra often struggle to understand the use of variables and, indeed, often resort to learning what are essentially symbolic tricks of various kinds that hold little meaning for them, such as regarding the symbols as letters rather than as objects of further significance (Booth, 1984). The numerical representation of a function offers other ways to think about the situation, and further explorations might be made to strengthen the meanings. Thus, Figure 15 shows how students can use the calculator to see that precisely the same table of results is generated by the function $f(x)=(x+2)(x+1)$ as by the original function, providing opportunities to understand the concept of factorizing an expression as well as deepening their understanding of the meaning of a variable, and experiencing the notions of identity and equivalence. Of course, students should explore these ideas in other ways as well as through the use of a calculator, including suitable visual representations of the relationships involved. 

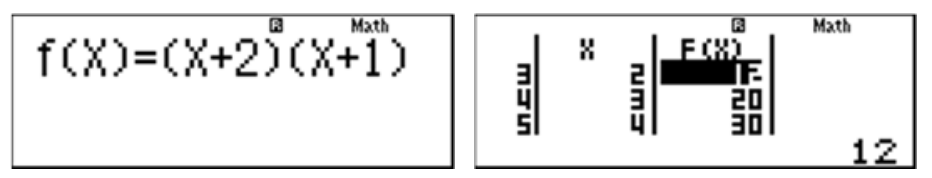

Figure 15. Representing $f(x)=x^{2}+3 x+2$ in factored form

More sophisticated students can use a calculator to explore relationships between indices and logarithms in a variety of ways. For example, Figure 16 shows some extracts from a table of logarithms to base 2, showing that the logarithms are larger for larger numbers and that some logarithms are integers. Students can also see from this activity that the logarithm of 6 is the sum of the logarithms of 2 and 3.


Figure 16. Exploring logarithms to base 2

The calculator permits direct exploration of the idea of a logarithm, as suggested by Figure 17, using logarithms to base 5, instead of base 2 . The primary idea that the logarithm of a number is the power to which the base needs to be raised to obtain the number can be seen on the calculator through direct manipulation, rather than via theoretical arguments, of the kinds that are often presented in textbooks to help students understand the key ideas.

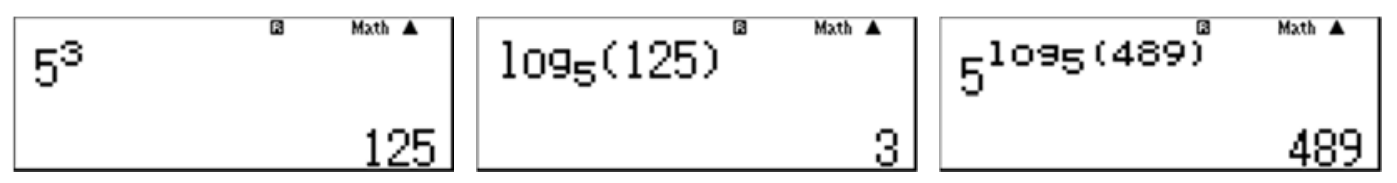

Figure 17. Exploring logarithms to base 5

In the senior secondary years, students can explore aspects of the calculus with a calculator that has numerical integrals and derivatives available. For example, numerical derivatives of the function $f(x)=x^{2}$ at a point can be efficiently obtained and compared, and interpreted as the slope of the associated curve at each point. With help, students can obtain several of these in succession, as shown in Figure 18.
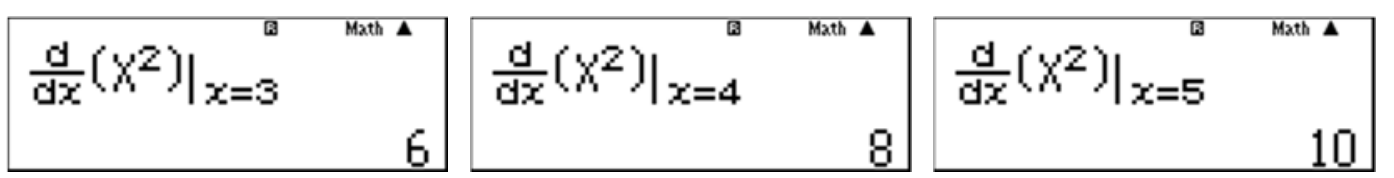

Figure 18. Numerical derivatives of $f(x)=x^{2}$ 
Through this means, and by looking for suitable patterns, students can build up the critical idea of a derivative function. While individual numerical derivatives can be interpreted as describing the slope of a function at a point, the relationship between them leads to the powerful idea that the function $f^{\prime}(x)=2 x$ describes all of these relationships together. Developing a sound grasp of this idea is crucial to understanding differential calculus.

In a similar way, starting with the idea of a numerical integral as describing the area under a curve between two points, students can explore suitable examples to see for themselves some of the properties of integrals. Figure 19 shows an example of this, with an integral of a sum of two functions having the same value as the sum of the two integrals.

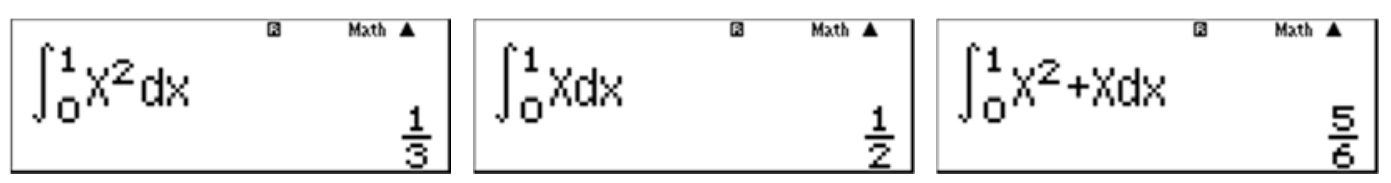

Figure 19. Exploring a property of integrals

Explorations of these various kinds are not intended to suggest that students should come to rely on their calculators to undertake the computations involved; rather, the scientific calculator is here used as a device to help students explore mathematical ideas, and it would be expected that they might later reach more sophisticated understanding of the ideas, as well as have more sophisticated explanations and arguments for them. The tool for learning is not intended to be the only way for students to learn, but rather is a powerful addition to existing methods, and helps to provide an environment that allow students to represent and explore ideas for themselves.

\section{Affirmation}

The final aspect of the model for educational use of calculators proposed by Kissane and Kemp (2014) concerns the use of calculators as devices for students to reassure themselves about their thinking. When students use a calculator thoughtfully, they should always have a sense of what to expect from a calculation, so that the dynamic nature of a calculator should always give rise to the possibility of affirmation (or, indeed, the possibility that their thinking will be contradicted by a surprising result of some kind). As a small example, a student who is confident that a number to two decimal places will be represented by a calculator as a fraction with a denominator of a hundred, will be affirmed by the first example in Figure 20, but at first possibly disconcerted or at least surprised by the following two examples. 

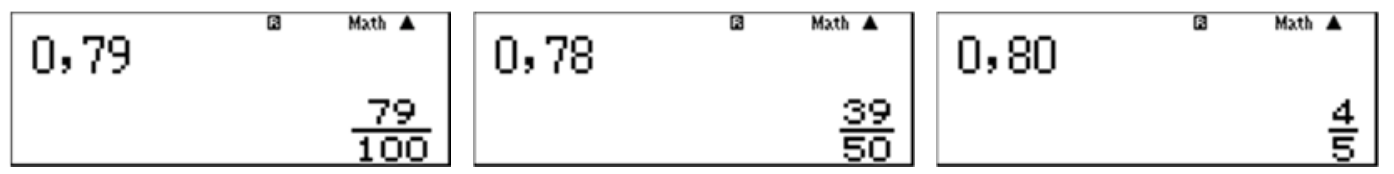

Figure 20. Anticipating fractions from decimals

As Jo Boaler (2016, p. 12) has observed, based on brain research, students can often learn more from mistakes than from correct answers, so that a device that allows them to test their thinking would seem to have some potential. In this case, while the students who expected $78 / 100$ and 80/100 respectively are not making mistakes, they are at least exposed to the need to think differently about the process of representing a decimal as a fraction, and thus have an opportunity to learn important lessons.

Developing such a thoughtful outlook is not always easy, however, and care is needed to encourage students to not merely use the device as an answering machine, but rather as an aid to thinking. On some calculators, opportunities to test one's thinking are inbuilt, opening up possibilities for learning from mistakes, as well as being reassured by correct predictions. The CASIO fx-991 ID PLUS, for example, has a Cek (check) facility, permitting students to get feedback on whether a particular mathematical statement is correct or not. Figure 21 shows an example of this being used, with the first line of each display showing a statement written by a student, and the second line showing the calculator's response of Salah (false) or Benar (true).
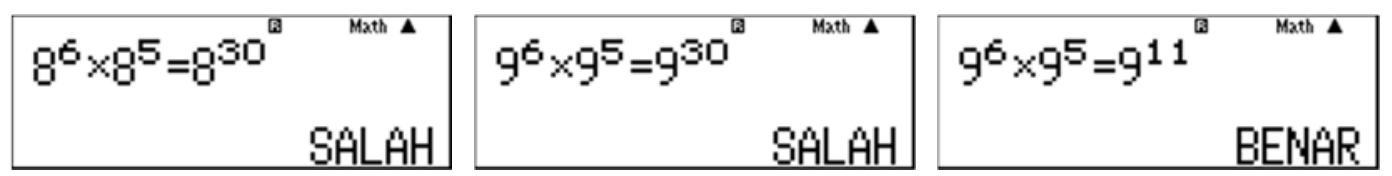

Figure 21. Using Cek mode to test predictions about indices

While it might seem natural for students to think that indices should be multiplied when indicial expressions are multiplied, more careful consideration suggests that they should be added, as reflected in the calculator's evaluations in Figure 21. The calculator is here providing feedback, with the student being confronted immediately with a recurring misconception, and an opportunity to learn from it.

Similarly, elementary algebraic errors, with which all teachers are familiar, can arise from students not fully understanding operations with variables. The first two screens in Figure 22 illustrate examples for which students can receive immediate feedback, advising them that 
there is something wrong with their thinking, while the third screen provides assurance in the form of an affirmation that their hypothesis is correct. Of course, students can learn much from this kind of experience if they are encouraged to discuss it with a partner, rather than working entirely by themselves.

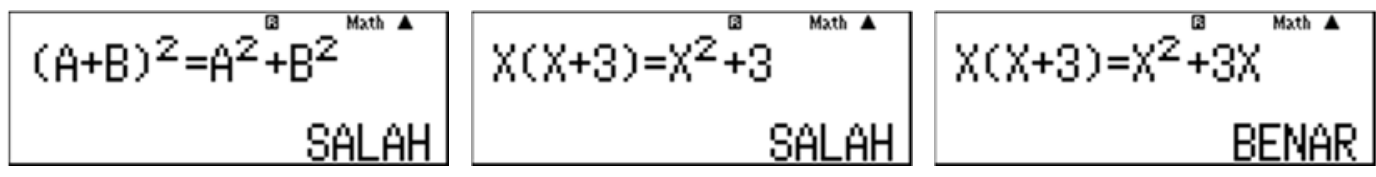

Figure 22. Encountering algebraic statements in Cek mode

In this case, the calculator is not using algebraic logic (as it does not have a computer algebra system), but is using the idea of a variable as represented by a calculator memory. Once again, these examples are not offered to suggest that students should rely on these kinds of mechanisms to evaluate expressions of these kinds. Rather, the calculator is a learning device that is both encouraging them and helping them to think about the meanings of the expressions, and to use the calculator as a feedback mechanism for affirmation (or contradiction). Other work, without a calculator, will build on this experience, and eventually the students would be expected to have strong formal understandings of the relationships involved, and to be able to both understand and generate formal proofs of them.

\section{Teachers and Pedagogy}

Scientific calculators are physical objects, and it is optimistic in the extreme to expect that their mere presence in a classroom will have substantial effect, without suitable intervention and management by teachers. In the recent study concerned with the use of computers in schools, the OECD (2015) observed that this is not an easy task:

\footnotetext{
One interpretation of these findings is that it takes educators time and effort to learn how to use technology in education while staying firmly focused on student learning. ... In the end, technology can amplify great teaching, but great technology cannot replace poor teaching. (p. 17)
}

Left to themselves, it seems likely that many students will merely regard the calculator as a means of answering numerical questions, and thus make limited use of the opportunities provided by this technology. Many of the suite of examples provided above of ways in which the calculator might impact upon students learning will not occur to students without some prompting, and a classroom environment in which intelligent calculator use is fostered and supported. So, it is important that the calculator be regarded as both a teaching tool and a learning tool, and that teachers receive adequate help to do so. 
In the first place, teachers need a good personal understanding of calculator capabilities, with an eye towards their potential benefits for education. Typically, calculator manuals offer advice on how to operate a calculator, but are much less likely to offer advice on the educational significance or even relevance of calculator operations. In any event, many users of calculators seem to misplace their manuals, which renders their effective educational use even less likely without some form of support. In countries in which calculators are not routinely expected for school use, limited help is typically provided by curriculum materials such as official guides and textbooks. Some online materials are available for those teachers with access to the Internet; Kissane and Kemp (2013) is an example of this sort of support.

Secondly, understanding how a calculator works is not enough for it to be used well for education purposes. Even where calculators are available, their use is often left to the final phases of lesson sequences, when students are completing exercises or problems for themselves, and require some computational support to do so. A change of mindset is often helpful, anticipating that a scientific calculator might be a useful learning device in earlier phases of lessons, and provide a means of experiencing mathematical ideas along with other materials in the everyday environment of a classroom. In this regard, Richardson (2014), responding to suggestions that calculators ought not to be used in English primary schools with young children, suggested that calculators ought to be part of the furniture of the classroom from an early age. She further noted that there was a strong case for the use of calculators in even the earliest years of schooling, supported by good research findings, but that "the full potential of calculators will only be realized with a skilled pedagogy" $(2014$, p.8) The case for the use of scientific calculators in secondary school is even stronger than the case for primary school, as substantially more of the mathematics curriculum can be encountered with a calculator, but the need for a skilled pedagogy is just as important.

If the scientific calculator is routinely available, and well understood by teachers, it can become part of the standard classroom environment. When their classroom has computer projection facilities, many teachers find an emulator version of a calculator a good classroom tool, as it can provoke public discussion of key ideas, as well as helping students to use their calculators well. However, the learning potential of the technology is maximized when individual students use their own calculators, often with direction, advice and support by the teacher. 
A recent publication (Kissane, 2016) offers some detailed suggestions for investigative tasks for students (in fact, pairs of students) using a modern scientific calculator (slightly different from that used in this paper). Such tasks may provide a helpful introduction to mathematical ideas and ways of approaching them through directed use of calculators. A key component of these tasks involves students working together, experimenting personally and explaining their observations to each other significantly more conceptually rich activities than merely obtaining answers to questions.

\section{Calculators and Curriculum}

As well as a skilled teacher, a supportive curriculum environment seems important for scientific calculators to have maximum possible educational impact. As the NCTM (2015) Technology Principle implies, effective use of technology depends in part on the mathematics that is included in the curriculum, as well as the ways in which it is taught.

There are many ramifications of this observation. For example, if a curriculum involves a study of probability and statistics, it would seem critical that it fosters the use of suitable technology to handle the data analysis involved and, in the case of probability, appropriate attention be given to methods of generating and using random data (e.g. for simulation purposes). Similarly, a mathematics curriculum that addresses the solution of equations should not be restricted to the (small) subset of equations that can be solved exactly, using by-hand methods; one would expect some attention to other forms of solution, such as iterative procedures or numerical approximations, when closed form solutions are not available. A curriculum that tried to engage students in mathematical modelling would seem to be problematic if it did not encourage the use of suitable technological support for exploring the relevance of particular models to practical data.

A key concept here is that of alignment between the teaching, learning and assessment program associated with a curriculum. In many Western countries, such as Australia, New Zealand, USA and European countries, scientific calculators (or frequently, graphics calculators, which are more powerful devices that have a graphics screen) are assumed to be routinely available for students to learn with and for teachers to teach with and are assumed to be routinely available during assessment as well. In some cases, partly to ease potential concerns about over-use of calculators, and partly to emphasise the importance of students undertaking mathematics at times with no external technology available to them, assessments include a calculator-free component, in addition to routine assessment for which students may 
use calculators if they so choose. Provisions of these kinds are not made for other forms of technology, such as computers, tablets and the Internet, since the challenge of ensuring reasonable and equitable access to these technologies for all students is still too great in all countries.

In designing mathematics curricula for the $21^{\text {st }}$ century, it seems problematic for many students to have no access to technology, but this is the likely outcome of technology use being regarded as optional. Curriculum developers need to make some assumptions regarding technology, and provide an environment in which those assumptions are accommodated by teachers, textbook publishers and examination authorities. As Kissane and Kemp (2012) argued, the most realistic assumption in developing countries, which often have a wide range of economic circumstances within them, is that a scientific calculator might be available to almost all students. A curriculum designed on the assumption that all students have access to some technology provides a kind of guarantee of minimal access for all. In that case, it becomes possible for some technology to be integrated into curricula, rather than being left only to a fortunate few.

\section{Conclusions}

This paper has outlined a case for more careful consideration being given to the potential benefits of scientific calculators in the mathematics curriculum. Although the name 'scientific calculator' suggests to some that the principle use of these devices is to calculate answers of a kind that scientists might need, a more careful consideration of a modern device suggests that its main significance may lie instead in mathematics education. At a time when many SEAMEO countries are keen to inject ICT into their curricula, and the available evidence suggests that this is a difficult task in practice, it seems timely to pay more careful heed to a technology that is small, portable, powerful and affordable and, unlike other forms of ICT, has been developed specifically to support secondary school mathematics, rather than being adapted from other purposes. Integrating scientific calculators successfully into schools requires some rethinking about the nature of the devices, adequate support for teachers to use them well, suitable complementary resources in the form of textbooks, and a curriculum and assessment environment that encourages students to use technology appropriately when it is necessary, and rely on analytical means when it isn't necessary. 


\section{References}

Boaler, J. (2016). Mathematical Mindsets. San Francisco, USA: Jossey-Bass.

Booth, L. (1984) Algebra: children's strategies and errors: a report of the Strategies and Errors in Secondary Mathematics Project. Windsor, NFER-Nelson.

National Council of Teachers of Mathematics (2015) Principles, Standards and Expectations. Reston, VA., NCTM.

Kissane, B. \& Kemp, M. (2014). A model for the educational role of calculators. Proceedings of the $19^{\text {th }}$ Asian Technology Conference in Mathematics (pp 211-220). Yogyakarta:

ATCM. Retrieved 14 June 2016 from http://atcm.mathandtech.org/EP2014/full/3672014_30001.pdf

Kissane, B. \& Kemp, M. (2012) The place of calculators in mathematics education in developing countries. Journal of Science and Mathematics Education in Southeast Asia, 35(2), 102-118.

Kissane, B. \& Kemp, M. (2013) Learning Mathematics with ES PLUS Series Scientific Calculator. Tokyo: CASIO. Retrieved 16 June 2016 from https://edu.casio.com/education/activity/

Kissane, B. (2016) Investigating Mathematics with ClassWiz. Tokyo: CASIO. Retrieved 16 June 2016 from https://edu.casio.com/education/activity/

OECD (2015), Students, Computers and Learning: Making the Connection, PISA, OECD Publishing. http://dx.doi.org/10.1787/9789264239555-en

Ronau, R., Rakes, C., Bush, S., Driskell, S., Niess, M. \& Pugalee, D. (2011) NCTM Research Brief: Using calculators for learning and teaching mathematics. Retrieved 29 July 2012 from http://www.nctm.org/news/content.aspx?id=31192

Richardson, G. (2014). Calculators - Challenging the rhetoric. Mathematics Teaching, July, 6-8. 